\title{
Prediction prices of Basrah light oil using artificial neural networks
}

\author{
Maysaa Abd Ulkareem Naser \\ Deprtement of Computer Information System, University of Basrah, Iraq
}

\begin{tabular}{l}
\hline Article Info \\
\hline Article history: \\
Received May 30, 2019 \\
Revised Nov 13, 2019 \\
Accepted Nov 26, 2019 \\
\hline
\end{tabular}

\section{Keywords:}

Artificial neural network

Feed fored neural network

Predication oil price

Recurrent neural networks

The reality of Iraqi oil

\begin{abstract}
The global economy is assured to be very sensitive to the volatility of the oil market. The beneficial of oil price collapse are both consumers and developed countries. Iraq's economy is a one-sided economy that completely depends on oil revenue to charge economic activity. Hence, the current decline in oil prices will produce serious concerns. Some factors stopped most investment projects, rationalize the recurrent outflow, and decreasethe development of the economic activity. The predicate oil prices are considered among the most complex studies because of the different dynamic variables that affect the strategic goods. The subject of forecasting has been extremely developing during recent years and some modern methods have been appeared in this regard, for example, Artificial Neural Networks. In this study, an artificial neural network (RFFNN) is adopted to extractthe complex relationships among divergent parameters that have the abilities to predict oil prices serving as an inputs to the network data collected in this research represent monthly time series data are Oil prices series in (US dollars) over a period of 11 years (2008-2018) in Iraq.
\end{abstract}

Copyright $(0) 2020$ Institute of Advanced Engineering and Science. All rights reserved.

\section{Corresponding Author:}

Maysaa Abd Ulkareem Naser,

Deprtement of Computer Information System,

University of Basrah,

The Site of Karma Ali, Iraq.

Email: maysaakarem13@gmail.com

\section{INTRODUCTION}

Iraq is exceptionally rich in oil but its economy suffers from severe structural failure. Iraq's oil reserves constant which amounted to about 107.313 billion barrels in 2018 are among the highest globally [1], In light of the existence of a financial economic crisis, the oil prices were severely affected by it, and although Iraq was not greatly affected by the crisis at its beginning as a result of the huge reserves of hard currencies that it achieved in the past years, specifically by selling oil during the great rise witnessed in these prices last period except it has been affected by the crisis, and oil prices have deteriorated recently and have become unstableMany economic analysts have confirmed that Iraqi oil sales will be affected by the global financial crisis strong until the crisis ends so the research aims to predict oil prices for the coming year and measure the extent of change in the coming period, Forecasting is one of the topics that are of great importance. Through forecasting, decision-makers can make the right decisions, [2].

Different influencing factors effect crude oil price changes. Complex dynamic interactions are among the factors that change crude oil prices and are one of the generality motivate and intriguing issues to be analyzed economically and financially through characterization and forecast characteristics. Recently, many experimental studies have shown the nonlinear nature of economic and pecuniary data, and it has been shown that conventional methods such as linear guessing methods cannot resolve the complex nonlinear dynamics concerned [3]. 
To predict oil price, there are three basic methods [4, 5]; the first one is based on the idea of optimal exploitation of resources, for example, Kurz [6] studied the petroleum prices from the "perspective of classical energy consumption". The second one, Robert [7] is studed the time series analysis method, Salah and Hamid [8] used the time series to analyze petroleum prices. While the third one is based on Ulph [9] through the exploration of exhaustible resources, that is, starting from the petroleum market structure. Wang et al [10] offered a crossbred methodology to predicate crude oil monthly prices. The paradigm consists of three groups discrete ingredients, Web mining from the authors debrief the rule-based system, aside from ANN, and ARIMA models. The three ingredients work disassembled and then intergraded with each other to get the results. In a related study, Lee and Teng [11] proposed that the RFNNs have the same advantages as RNNs and expanded the application field of the FNNs to temporal problems. Wang [12] used the recurrent neural network (RNN) to predicat the crude oil pointers. Chiroma et al [13] suggested a genetic algorithm enhanced neural network model to predicate the crude oil price inconstancy. They indicated this evolutional neural network model brings statistically important performance amelioration. Chiroma et al [14] provided a wipe inclusive on the AI and ML-based crude oil predicating models. Brooks et al [15] deliberate the lead-lag relation on high-waver data $10 \mathrm{~min}$,for FTSE index. The authors proposed the lead-lag 4 relations could only contract for no more than 30 minutes, and their results proposed variable in futures prices may help to predicat the changes in the instant price.From previous studies, we find that the neural networks are better for predicting oil prices for the purpose of grasping efficiently and accurately the tendency and regularity of oil price change and in view of the influencing factors of petroleum price, the artificial neural network is commonly used to solve complex nonlinear problems [16].

We propose a neural model in which ANNs have been extensively applied for many assignments, like non-linear control, sample classification, assignment approximation, time series forecasts, and effort or costs forecasts $[17,18]$. FFNNs are the simplest and first kind of ANNs. In FFNNs, the data always takes just a single direction, from the inputs nodes, during hidden nodes then to the outputs nodes and no loop in those networks. These networks employ a lot of neurons (node) and have no feedback path within. They are vastly used NNs, practically in systems and control. Multilayer NNs have input-layer, hidden-layers, and finally, output-layer, "no interconnections between nodes at same layers" as shown in Figure 1 [19], they are called (hidden) because of the internality to the network and present between network layers, input, and output layer [20]. Training Algorithm of FFNN error-training algorithm feedback is a learning-control algorithm of ANNs, it calculates the gradation of the error function pertains the weights of the neural network. However, it`s considered as a popularization of delta rule of the perceptron to a multi-layer FFNNs [21].

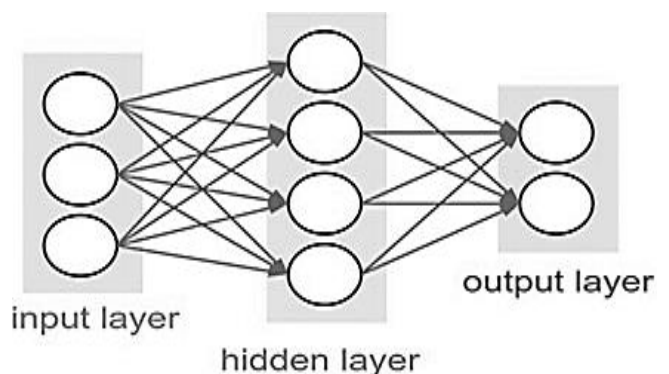

Figure 1. Feedforward neural network (FFNN)

ANNs with recurrent connections are called recurrent neural networks (RNNs), which enable modeling consecutive data for consecute and forecasts [22]. Recurrent neural networks (RNNs) were developed originally by Hopfield in a single-layer form; and after a while, these networks were developed applying a multilayer perceptron involved connected input-output, processing, and output layers [23, 24]. Having an importance over standard FFNN, RNN can transact with sequential input data, using their internal memory to process sequences of inputs and use the formerly stored information to assist in making future forcasting. This is done by feedback connections or by loops exist between neurons, which authorize them to be more complex data forecasts $[25,26]$. The network structure is in some way similar to the multilayer perceptron, with some discrepancies that permit connection happens through hidden units that are associated with delays in time. These models can save information of past times through such this connection. It is NNs continuously operate depending on time. RNNs accept input vectors at every time step, also update its hidden states with non-linear activation functions, and after that uses it to make imagination and forcasts for its outputs through making the output value feebdback to input layer as a new input in the input layer or the hidden layer 
value return to the input layer as a recurrent mode $[27,28]$. Recurrent neural networks (RNNs) have been proven as an active tool to paradigm timing -dependency in diffrent applications.

In this work, we offer a RNN model for the crude oil price, FFNN also provides "the flexibility and complexity to come close to non-linear functions to any preferred accuracy by changing number of layers and hidden neurons of each respective layer". FFNN is adopted to upgrade a model for the proposed method RFFNN recurrently to forcecast oil value. The focus of this paper is to apply neural networks to predicate crude oil future prices. The objectives: predicate crude oil future prices applying RFNNs, and find how training time can affect on accuracy of predicate. The RFFNN is better from normal FFNN to FFNN in a recurrent mode to give the network capability to make predication for the future not only the predicate of the current time. The paper is arranged as follows. Two kinds of artificial neural networks are described in Section 1. Section 2 The execution of the proposed learning algorithms is tested by the computer simulation software and predicate results are detailed in Section 3. The conclusion is presented in Section 4.

\section{RESEARCH METHOD}

\subsection{Experimental data}

The inputs of the RFFNN proposed must be selected depending on which input data affects the predication. Data collected in this research represent time series data monthly which are oil prices series in (US dollars) during the period starting from January 1, 2008 to April 30, 2018 in Iraq [29, 30]. As shown in Table 1.

Table 1. The monthly consumption of oil price in the Basrah-Iraq (2008-2018)

\begin{tabular}{|c|c|c|c|c|c|c|c|c|c|c|c|}
\hline \multirow[t]{2}{*}{ Month } & \multicolumn{11}{|c|}{ Year } \\
\hline & 2008 & 2009 & 2010 & 2011 & 2012 & 2013 & 2014 & 2015 & 2016 & 2017 & 2018 \\
\hline Jan & 84.70 & 33.91 & 70.74 & 90.776 & 109.081 & 105.87 & 102.70 & 42.25 & 25.01 & 46.71 & 62.596 \\
\hline Feb & 86.20 & 33.38 & 72.84 & 98.442 & 122.928 & 104.73 & 103.38 & 51.82 & 27.51 & 51.07 & 60.060 \\
\hline Mar & 96.40 & 47.11 & 77.76 & 107.13 & 177.994 & 104.33 & 102.10 & 50.533 & 28.05 & 46.99 & 60.114 \\
\hline Apr & 99.80 & 47.11 & 79.05 & 114.361 & 166.799 & 100.31 & 102.11 & 55.61 & 36.62 & 53.78 & 64.889 \\
\hline May & 113.50 & 58.69 & 71.41 & 108.261 & 103.039 & 100.31 & 103.16 & 60.40 & 42.05 & 53.07 & 69.801 \\
\hline Jun & 121.30 & 61.00 & 71.41 & 105.176 & 90.097 & 99.30 & 105.80 & 58.63 & 44.63 & 51.51 & 68.758 \\
\hline Jul & 122.30 & 64.60 & 69.92 & 108.795 & 97.141 & 97.05 & 103.83 & 53.10 & 41.37 & 49.83 & 68.566 \\
\hline Aug & 104.10 & 66.03 & 69.00 & 104.919 & 106.226 & 101.32 & 99.20 & 44.32 & 42.01 & 48.50 & 69.651 \\
\hline Sep & 94.80 & 66.03 & 71.33 & 104.897 & 107.596 & 101.32 & 94.49 & 43.41 & 41.88 & 50.91 & 74.159 \\
\hline Oct & 68.00 & 74.16 & 76.62 & 104.043 & 105.51 & 99.86 & 83.57 & 43.50 & 46.79 & 52.08 & 73.348 \\
\hline Nov & 51.50 & 73.14 & 79.94 & 106.599 & 104.326 & 100.01 & 73.57 & 38.70 & 41.97 & 53.01 & 61.150 \\
\hline Dec & 37.30 & 74.31 & 79.94 & 106.18 & 103.723 & 104.11 & 57.94 & 32.06 & 50.87 & 55.04 & 53.962 \\
\hline
\end{tabular}

\subsection{Suggested model}

The idea for designing the recurrent feed-forward neural network (RFFNN) is to predict oil prices. RFFNN includes 12 nodes in the input layer making the first stage, 6 hidden-layer nodes specific by experiment and error for stability, 1 node in the output layer. The method RFFNN proposed recurrently uses FFNN for predicting oil prices. RFFNN is shown in Figure 2.

- $\quad$ The inputs (M1, M2, M3..., M12) of the suggest RFFNN must be selected dependent on which parameters are affecting on prediction, the parameters that were used in this proposed method are 12 parameters data on a monthly basis which are oil prices series during the period starting from January 1, 2008 to April 30, 2018 in Iraq.

- $\quad \mathrm{R} 1 \ldots \mathrm{RN}$, represents network outputs that are reused as inputs to the network.

- The data entered were randomly divided into three groups: training group, examination group and test group, $70 \%$ for the training group and $15 \%$ for the verification and testing groups

- The training was chosen as Representative of the reverse training algorithm Backpropagation. The learning rate is 0.01 and training them, are the most widely used and used in network training FFNN because they have a high speed of reaching the best solution.

- Determine the number of neurons in the hidden layer by experimentation through changing the number of neurons in this layer and the train network to achieve the optimal performance standards for the network trained. 


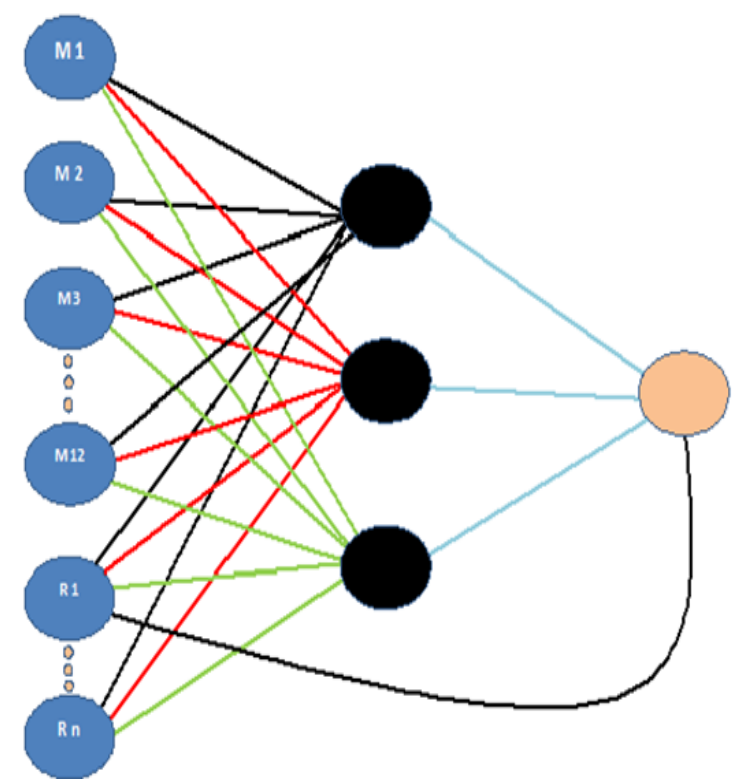

Figure 2. Structure of the proposed (RFFNN)

\subsection{Working method}

In this paper, the network works in the following way, the training algorithms and the suggested RFFNN test are designed with $\mathrm{C}$ programming language Uses a backpropagation learning algorithm. suggested RFFNN trains on 20 various modes. Training patterns Contains input as $12,13 \ldots$ and 16 parameters and output is required to estimate oil prices in the training phase, 0.9 workers are training.

A 0.6 homogenization factor is used which is selected by experiment and wrong to give better convergence and fewer epochs.

The following weighted-average equations -used to compute the required output:

$$
X i=P S i * S i
$$

$$
\begin{aligned}
& Z i=P S U M i / X i \\
& W i=P E R i * Z i \\
& E=\sum W_{i}
\end{aligned}
$$

where,

PS: number of parameters in each class.

$\mathrm{S}$ : number of scales that the parameters

PSUM: summation of all scales in each class.

W: class weight.

E: computed prediction

$$
P E R=\left(\frac{P S}{\text { Total number of parameter }}\right) * 100
$$

where,

PER: the ratio of every class where, the first class is $70 \%$, the second class is $15 \%$ and third class is $20 \%$. The flowchart of the proposed RFFNN illustrated in Figure 3. 
The flowchart of the proposed RFFNN illustrated in Figure 3.

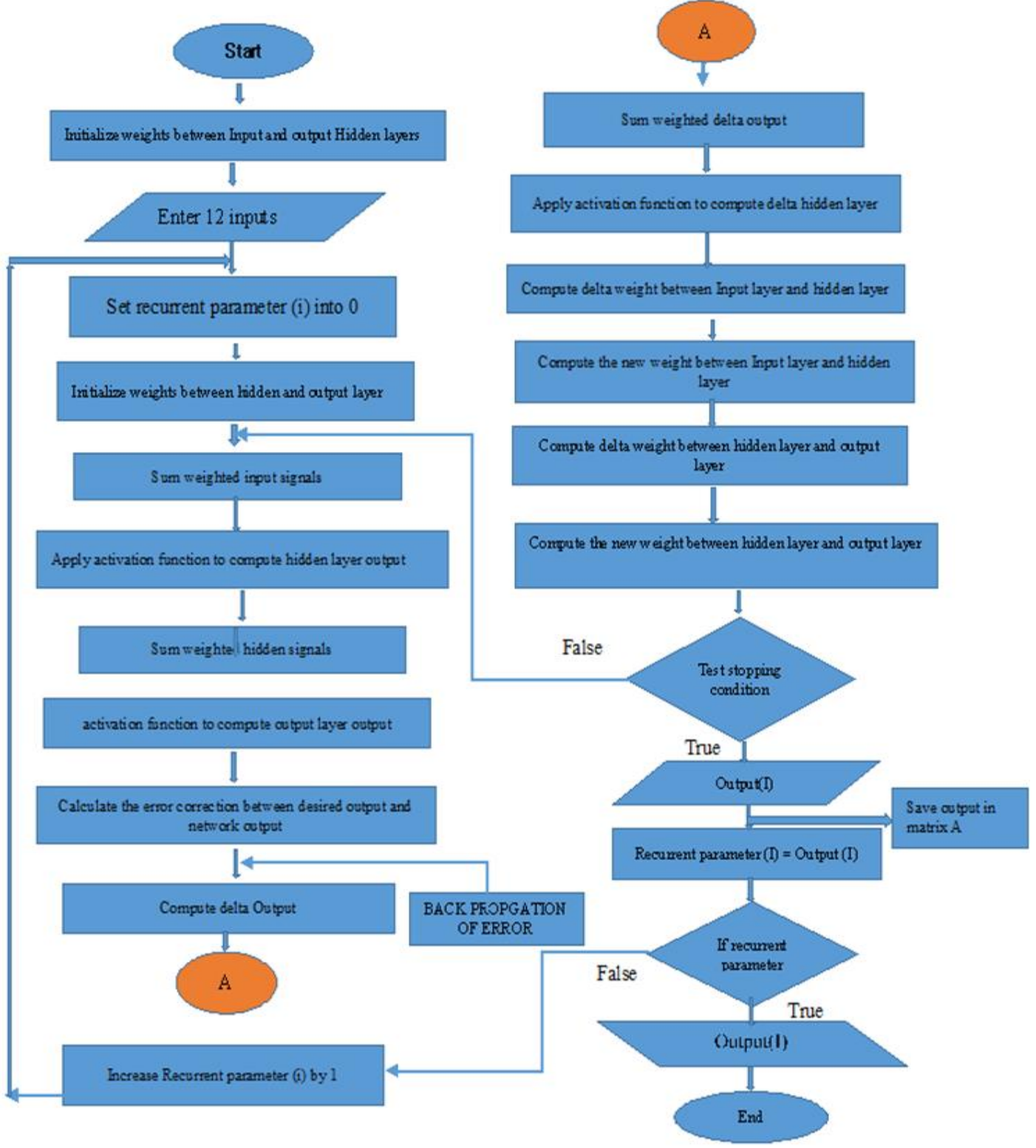

Figure 3. The flowchart of the proposed (RFFNN) for predication crude oil price in Basrah city

\section{SIMULATION RESULTS AND DISCUSSIONS}

To demonstrate the performance of the proposed RFFNN scheme as shown in Figure 3, designed using $\mathrm{C}++$ programming language and backpropagation learning algorithm is used.Points can be clarified phase means one training course.

- Training phase 1 , which is 80.20 , is stored in the matrix and becomes Additional inputs for the training phase 2.

- Training Phase 2 outputs are 75.9 with Output phase 1 which is 80.20 becomes additional input in the training phase 3 .

- The output of training Phase 3 which is 76.76 with the previous outputs of training stage 1 and 2 become additional inputs in training Phase 4.

- The output of training Phase 4 which is with the outputs of training phase 1,2 , and 3 become additional inputs in training Phase 5.

- Thus, network works for 12 entries in each process of guessing. 
The output at stages $\mathrm{N}-1, \mathrm{~N}-2, \ldots$ and 1 of the proposed RFFNN that works in a recurrent fashion are entered as inputs of stage N. Recurrent parameters are influenced to train the network for forecasting of the oil price. Table 2 and Figure 4 show the results of RFFNN of the forecast monthly oil price of Basrah city for (2019). Table 3 shows the evaluated RFFNN, using the following equations, where the results of the error using the magnitude of relative error (MRE) and the mean magnitude of relative error (MMRE) equations are very small values, which are close to zero.

MRE is a criterion that is used to evaluate the predicted oil price. The MRE obtained as [31]:

$$
M R E=\left[\frac{X 1_{\text {actual }}-X 2_{\text {predicted }}}{X 1_{\text {actual }}}\right]
$$

where,

$\mathrm{X}_{1}$ : is the actual predicted.

Predicted: is the predicted oil price.

The Mean Magnitude of Relative Error:

MMRE is obtained from the summation of MRE over $N$ observations [32].

$$
M M R E=\frac{\sum_{i=1}^{N} M R E}{N}
$$

where,

$\mathrm{N}$ : is the number of projects for which is predicted

where the results of the error equation are a very small value.

Table 2. Predication monthly oil price of Basrah city for 2019

\begin{tabular}{ccccccccccccc}
\hline Month/Predicate & Jan & Feb & Mar & Apr & May & Jun & Jul & Aug & Sep & Oct & Nov & Dec \\
\hline & 80.20 & 75.91 & 76.76 & 76.61 & 77.61 & 78.47 & 79.32 & 81.18 & 76.20 & 75.91 & 76.83 & 77.61 \\
\hline
\end{tabular}

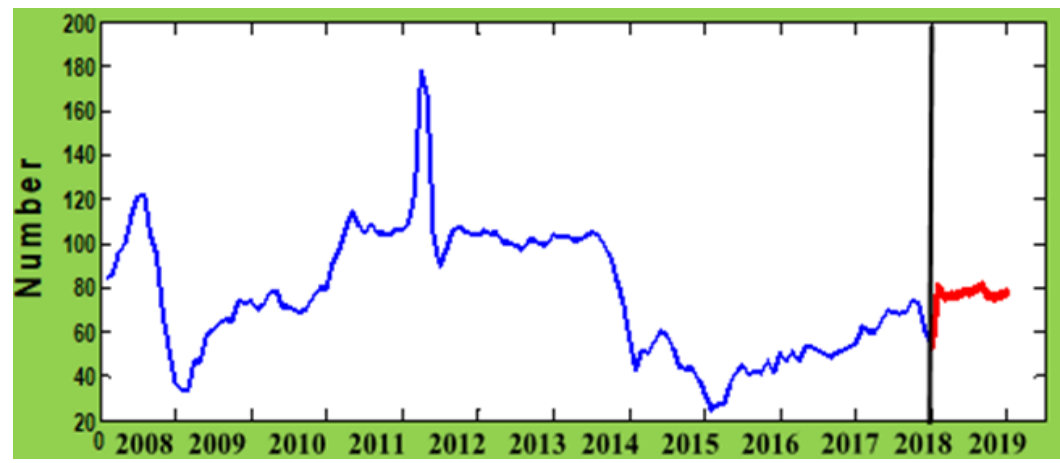

Figure 4. Prediction results of monthly oil price of Basrah city for (2008-2019)

Table 3. The represents its error value during network training

\begin{tabular}{ccc}
\hline Model/Error Value & MRE & MMRE \\
\hline & 0.024 & 0.016 \\
\hline
\end{tabular}

\section{CONCLUSION}

The oil sector is the main source for the supply of foreign currency needed by Iraq in the development plan and to cover its needs of imported goods and the need of commodity sectors of raw materials and the rest of supplies to sustain the national production, and from this point of view we have to build a neural network that has the capability to predict the future oil prices for Iraq. In this research neural network approach has shown a strong predictive capability, to give the network ability of prediction of future not only the prediction of the current time. The methods for oil price prediction by implementing FFNNs and during it find the better parataxis between required and current outputs are gained. the RFFNN is improved from normally, FFNN to FFNN in a recurrent style prediction process that has a considerable function in the execution of Oil price predicate, affected by execution time, data size, debugging capability. They are influenced relatively where 
the RFFNN is used for oil price forecasting In the suggested RFFNN, it is perceived that the forecasting of future oil price prediction depended on analogous oil price predictions in previously mentioned stages.RFFNN using the rubrice of time series to make forecasting the output of time $(\mathrm{t})$ returns as input in time (t-1). This used for improve the FFNN to FFNN in a recurrent modality.crude oil market is the most volatile commodities market. Therefore, the predicted oil price using nonlinear models is a right option. As future work, another kind of smart procedure can be used with neural networks and reinforce such as crossbred systems like

"Neuro-Fuzzy system" and as well as RFFNN can be optimize design by using genetic algorithms.

\section{REFERENCES}

[1] Oil market report, international energy agency, 2018, https://www.iea.org/oilmarketreport/reports/.

[2] Al-Bayan Center for Planning and Studies, "Oil and gas report, including forecasts for the next ten years until the year 2025," Iraq, Nov. 2016.

[3] F. Shen, et al., "Forecasting exchange rate using deep belief networks and conjugate gradient method," Neuro computing, vol. 167, pp. 243-253, 2015.

[4] S. Wlazlowski, et al., "Price dynamics in European petroleum markets," Energy Economics, vol. 31, no. 1, pp. 99-108, 2009.

[5] C. Hendrickson, "Petroleum prices and transportation engineering," Journal of Transportation Engineering-asce, vol. 134, no. 9, pp. 359-360, 2008.

[6] H. D. Kurz and H. Salvadori, "Classical economics and the problem of exhaustible resources Metro economic," vol. 52, no. 3, pp. 282-296, 2001.

[7] P. Robert, "The long-run evolution of energy prices," The energy journal, vol. 20, no. 2, pp. 1-27, 1999.

[8] S. Abosedra, H. Baghestani, "On the predictive accuracy of crude oil future prices," Energy Policy, vol. 32, no. 12, pp. 1389-1394, 2004.

[9] M. Ulph and G.M. Folie, "Exhaustible resource and cartels: an extemporal nash-cournot model," The Canadian journal of economics, vol. 13, no. 4, pp. 645-658, 1980.

[10] S. Wang, et al., "Crude oil price forecasting with TEI@ I methodology," Journal of Systems Science and Complexity, vol. 18 , no. $2,145-166,2005$.

[11] C.H. Lee and C.C. Teng, "Identification and control of dynamic systems using recurrent fuzzy neural networks," IEEE Transactions on Fuzzy Systems, vol. 8, no. 4, pp. 349-366, 2000.

[12] J. Wang, "Forecasting energy market indices with recurrent neural networks: Case study of crude oil price fluctuations," Energy, vol. 102, no. 2016, pp. 365-374, 2016.

[13] H. Chiroma and S. Abdulkareem, T. Herawan, "Evolutionary neural network model for west texas intermediate crude oil price prediction," Applied Energy, vol. 142, no. 2015, pp. 266 - 273, 2015.

[14] H. Chiroma, et al., "A review on artificial intelligence methodologies for the forecasting of crude oil price," Intelligent Automation and Soft Computing, vol. 22, no. 3, pp. 449-462, 2016.

[15] Brooks, et al., "A trading strategy based on the lead-lag relationship between the spot index and futures contracts for the FTSE 100," International Journal of Forecasting vol. 17, pp. 31-44, 2001.

[16] H. Song, et al., "Power Transformer Operating State Prediction Method Based on an LSTM Network," 2017 IEEE International Conference on Prognostics and Health, vol. 41, no. 23, pp. 156-167.

[17] N. I. Al-Bulushi, et al., "Artificial neural networks workflow and its application in the petroleum industry," Neural Computing \& Applications, vol. 21, no. 3, pp. 409-421, 2012.

[18] Q. J. Ziang, K.C. Gupta, V. K. Devabhaktuni, "ANN for RF and Microwave Design-From theory to practice," IEEE Transactions on Microwave Theory and Techniques, vol. 51, no. 4, pp. 1339-1350, Apr. 2003.

[19] M. Hagan, et al., "Neural Network Design," Bost. Massachusetts PWS, vol. 2, pp. 734, 1995.

[20] M. Kubat, "Neural networks: a comprehensive foundation," The Knowledge Engineering Review, vol. 13, no. 4. pp. 409-412, 1999.

[21] A. Madadlou and Z. Emam-Djomeh, "Surface optimization of an artificial neural network for predicting the size of re-assembled casein micelles," Compute. Electron Agric., vol. 68, pp. 216-221, 2009.

[22] M. Liang and X. Hu, "Recurrent convolutional neural network for object recognition," in 2015 IEEE Conference on Computer Vision and Pattern Recognition (CVPR), Boston, MA, pp. 3367-3375, 2015.

[23] Y.C. Hu and R.R.W. Lin, "Applying Neural Networks to Prices Prediction of Crude Oil Futures," Article in Mathematical Problems in Engineering, Aug. 2012.

[24] J. Martens and I. Sutskever, "Learning recurrent neural networks with Hessian-free optimization," Proc. 28 th Int. Conf Mach. Learn. ICML 2011, pp. 1033-1040, 2011.

[25] A. ElSaid, F. El Jamiy, et al., "Optimizing long short-term memory recurrent neural networks using ant colony optimization to predict turbine engine vibration," Applied Soft Computing, vol. 73, pp. 969-991, Dec. 2018.

[26] J. Martens and I. Sutskever, "Learning recurrent neural networks with Hessian-free optimization," Proc. 28 th Int. Conf. Mach. Learn. ICML 2011, pp. 1033-1040, 2011.

[27] R. Pacanu et al., "On the Difficulties of Training Recurrent Neural Networks," ICML, no. 2, 2013.

[28] S. Kulkarni and I. Haidar, "Forecasting Model for Crude Oil Price Using Artificial Neural Networks and Commodity Futures Prices," International Journal of Computer Science and Information Security, vol. 2, no. 1, Jun. 2009.

[29] OAPEC, Annual Bending Report, Arab Countries Organization, Mandara Petroleum, Kuwait, Different Years. 
[30] "Annual Statistical Report of Al Basrah Oil Company," Ministry of Oil, Iraq, 2018, [Online]. Available: https://oil.gov.iq/

[31] Idri, et al., "RBFN Networks- based Models for Estimating Software Development Effort," A Cross- validation Study 2015 IEEE Symp. Ser. Comput. Intell., vol. 39, no. Ml, pp. 976-983, 2015.

[32] M. Bisi and N.K. Goyal, "Software development efforts prediction using artificial neural network," IET Inst. Eng. Technol., vol. 10, no. 3, pp. 63-71, 2016.

\section{BIOGRAPHY OF AUTHOR}

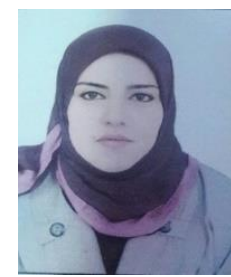

Maysaa Abd Ulkareem Naser is currently a lecturer in Department of Computer Information System, College of Computer Science and Information Technology, University of Basrah - Iraq. She received the bachelor"s degree in Computer Science from Basrah University, Iraq in 1998. In 2001, she received the master"s degree in Computer Science from Basrah University. Her main research interests include, intelligent system, Natural Language Processing 